ARTICLE

\title{
Glycogen controls Caenorhabditis elegans lifespan and resistance to oxidative stress
}

Ivan Gusarov¹, Bibhusita Pani ${ }^{1}$, Laurent Gautier ${ }^{1}$, Olga Smolentseva1, Svetlana Eremina², Ilya Shamovsky ${ }^{1}$, Olga Katkova-Zhukotskaya ${ }^{2}$, Alexander Mironov ${ }^{2}$ \& Evgeny Nudler ${ }^{1,3}$

A high-sugar diet has been associated with reduced lifespan in organisms ranging from worms to mammals. However, the mechanisms underlying the harmful effects of glucose are poorly understood. Here we establish a causative relationship between endogenous glucose storage in the form of glycogen, resistance to oxidative stress and organismal aging in Caenorhabditis elegans. We find that glycogen accumulated on high dietary glucose limits C. elegans longevity. Glucose released from glycogen and used for NADPH/glutathione reduction renders nematodes and human hepatocytes more resistant against oxidative stress. Exposure to low levels of oxidants or genetic inhibition of glycogen synthase depletes glycogen stores and extends the lifespan of animals fed a high glucose diet in an AMPK-dependent manner. Moreover, glycogen interferes with low insulin signalling and accelerates aging of long-lived daf- 2 worms fed a high glucose diet. Considering its extensive evolutionary conservation, our results suggest that glycogen metabolism might also have a role in mammalian aging.

\footnotetext{
${ }^{1}$ Department of Biochemistry and Molecular Pharmacology, New York University School of Medicine, New York, New York 10016, USA. ${ }^{2}$ Engelhardt Institute of Molecular Biology, Russian Academy of Science, Moscow 119991, Russia. ${ }^{3}$ Howard Hughes Medical Institute, New York University School of Medicine, New York, New York 10016, USA. Correspondence and requests for materials should be addressed to E.N. (email: evgeny.nudler@nyumc.org).
} 
A ll organisms employ similar pathways of glucose utilization and storage. Dietary carbohydrates are converted to glucose, which eventually donates its carbons to most other synthesized biomolecules. Glucose also serves as a major energy source (ATP production) and fuel for recycling reducing equivalents (NADPH and glutathione (GSH)). Surplus glucose is stored as glycogen or triglycerides for future energy needs. However, sugar overconsumption is uniformly detrimental to animals, as it leads to excessive body weight ${ }^{1}$, early onset of many disorders ${ }^{2,3}$ and reduced lifespan ${ }^{4,5}$. In contrast, caloric restriction extends the lifespan of both worms and mammals ${ }^{6-8}$. Despite extensive research, the fundamental mechanisms underlying the detrimental effects of glucose have not been well established.

Caenorhabditis elegans is an excellent model organism in which to study the effects of glucose on animal physiology and aging $^{9-12}$. It has been proposed that activation of the insulin/ insulin-like growth factor-1 signaling (IIS) pathway is the cause of reduced lifespan in worms fed a glucose-rich $\operatorname{diet}^{13}$. While long-lived mutant (daf-2) with reduced IIS also exhibited a short lifespan if exposed to glucose ${ }^{13}$, arguing that it is sensitive to a high glucose diet. Recent studies implicated the increased lipid production in worms' tolerance to a high glucose diet, indicating that the lowering of cellular glucose by converting it to lipids can be beneficial ${ }^{9}$. Also, accelerated degradation of the products of glucose catabolism renders worms resistant to high glucose ${ }^{10}$. Thus the activation of any glucose-utilization pathway appears to be beneficial for the lifespan ${ }^{9,10}$.

The elevated level of reactive oxygen species (ROS) has been associated with a high glucose diet, albeit only in very old animals ${ }^{10}$, suggesting that in younger animals that may not be the case. Chemical inhibition of glucose metabolism also increased ROS formation and extended the nematode lifespan ${ }^{11}$. The traditional view of ROS as damaging agents responsible for the aging process has gradually been revised ${ }^{4,15}$, and it is now widely accepted that a certain level of ROS is necessary for normal cellular function and actually promotes longevity ${ }^{16-18}$. Sublethal doses of oxidants, or elevated endogenous ROS, were shown to extend the C. elegans lifespan ${ }^{18-21}$. In contrast, antioxidants often fail to influence lifespan and may actually accelerate aging $19,21,22$. We used C. elegans to examine the mechanism and role of ROS in glucose-mediated life shortening.

Here we show that glycogen, accumulated on a high glucose diet, drives cellular thiol reduction and thereby protects C. elegans and human hepatocytes against oxidative stress. In contrast, high glycogen depot shortens worm's lifespan. Knocking down glycogen synthase or using oxidants to reduce glycogen stores extends lifespan of C. elegans in AMP-activated protein kinase (AMPK)-dependent manner. The evolutionary conservation of glycogen metabolism suggests that the glycogen-mediated impact on cellular redox status and aging might be universal.

\section{Results}

Glucose renders C. elegans resistant to oxidative stress. Typically, resistance to oxidative stress correlates with longevity ${ }^{23}$. For example, long-lived daf-2 are more resistant to oxidants than are wild-type (wt) worms, and short-lived daf-16 are sensitive ${ }^{24-26}$. Surprisingly, however, we found that a high glucose diet, which substantially shortens the C. elegans lifespan (Fig. 1b) ${ }^{10,11,13}$, also greatly protects worms against oxidants, such as diamide and paraquat (Fig. 1a). This observations seems even more surprising, as earlier microarray studies did not detect induction of any known oxidative stress genes in response to glucose ${ }^{13}$. This is, however, supported by another observation of glucose-induced resistance to a different oxidant, juglone ${ }^{27}$. Moreover, expression of the oxidative stress marker gene, sod-3::GFP, was suppressed by glucose ${ }^{13}$. Many cytoprotective genes including sod-3 are regulated by the IIS-dependent transcription factor DAF-16. Because glucose also protects daf-16 knockdown worms against diamide (Fig. 1a), we conclude that the antioxidant effect of glucose is not mediated by daf- 16 .

We next examined whether glucose also protected C. elegans against chronic exposure to an oxidant. As expected, a high level of diamide shortened the lifespan, whereas glucose abrogated this effect (Fig. 1b, Table 1). Taken together, these results demonstrate that glucose helps the animals to cope with oxidative stress.

Glucose does not induce oxidative stress response in worms. It has been proposed that glucose consumption increases ROS in very old worms ${ }^{10}$. Hypothetically, elevated ROS on a high glucose diet may induce detoxifying genes, which would render animals more resistant to oxidative stress. To investigate this, we monitored the changes in global gene expression in response to glucose. RNA-seq differential expression analysis revealed 168 and 58 upregulated and downregulated genes, respectively, after 1 day of glucose feeding (Fig. 1c, Supplementary Table 1). As expected, glucose induced significant metabolic changes (Fig. 1c). About $20 \%$ of differentially regulated genes are involved in lipid metabolism. Amino acid and polyamine degradation genes are suppressed, whereas glutamine, asparagine, S-adenosylmethionine and glycerol-3-phosphate biosynthesis are upregulated. These results indicate that sugar consumption promotes net amino acid biosynthesis, sphingolipid and phospholipid metabolism and lipid storage in the form of triglycerides (Supplementary Table 1). Indeed, in agreement with previously published data, glucose-fed worms accumulated more lipids

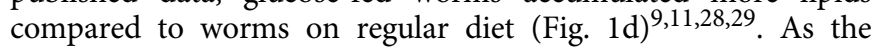
elevated lipid storage is a hallmark of sugar overconsumption in mammals, C. elegans can be viewed as a relevant proxy model to study the mechanism of glucose toxicity. Interestingly, the majority of downregulated genes are related to immune response (Supplementary Table 1). However, since glucose shortens the lifespan of $C$. elegans on either live or killed Escherichia coli or on non-pathogenic B. subtilis, its toxicity cannot be attributed to enhanced pathogenicity ${ }^{13,30}$.

Notably, the list of genes upregulated by glucose is not enriched with ROS-detoxifying or ROS-damage repair genes (Supplementary Table 1). Out of the 115 genes known to be involved in the oxidative stress response (Worm Base gene ontology term GO:0006979), only one hypothetical thioredoxine-like gene (W01B11.6) was modestly induced by glucose. Thus glucosemediated ROS resistance (Fig. 1a) cannot be explained by hormesis and upregulation of protective genes, but instead it appears to activate a pre-existent defense mechanism.

Glucose-mediated GSH reduction protects against oxidants. With the exception of ROS-neutralizing enzymes, GSH acts as the major cellular defense against oxidant ${ }^{31}$. For example, after oxidation to disulfide (GSSG), it is re-reduced to GSH by glutathione reductase (GSR), which uses NADPH as the electron donor ${ }^{32}$. The pentose phosphate pathway (PPP) generates most of the cellular NADPH from glucose-6-P (Fig. 2c). Thus surplus glucose should accelerate GSSG reduction and increase oxidant scavenging. Indeed, we detected an increase of cellular reduced thiols in worms fed a high glucose diet (Fig. 2a). Moreover, the glucose-fed worms maintain their level of reduced thiols in response to diamide much better than worms on a regular diet (Fig. 2a). To further implicate PPP in glucose-mediated resistance to oxidants, we used RNAi to knockdown glucose-6-P dehydrogenase (gspd-1) or GSR (gsr-1) (Fig. 2c). Worms lacking either of these enzymes became 
a

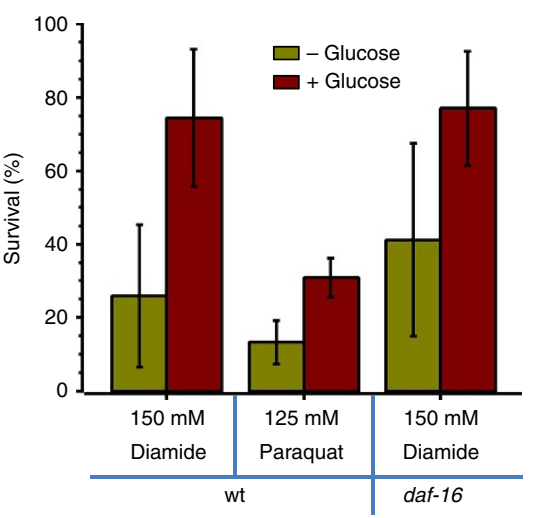

C

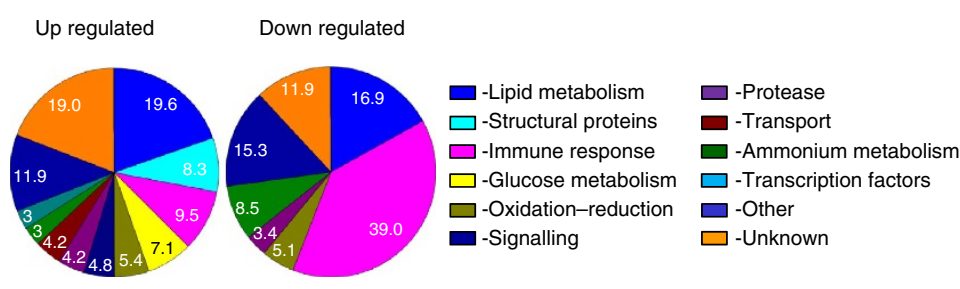

b

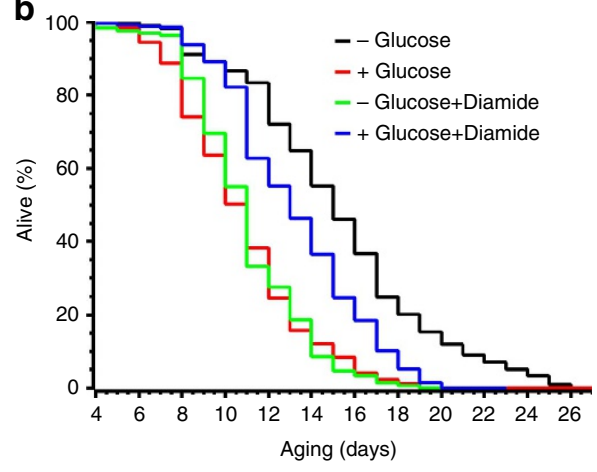

d

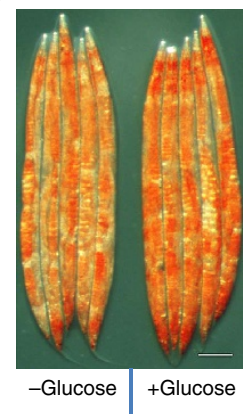

Figure 1 | Dietary glucose protects C. elegans from oxidative stress. (a) Dietary glucose protects wt and daf-16 worms against $125 \mathrm{mM}$ paraquat ( $n=190$, $P=0.0006$ ) or $150 \mathrm{mM}$ diamide (wt: $n=170 P=0.0057$; daf-16: $n=140, P=0.0284$ ). L4 stage worms were placed on NGM plates with sorbitol (green) or glucose (red) for $\sim 20 \mathrm{~h}$ at $20^{\circ} \mathrm{C}$ and then treated with the indicated oxidants. To compensate for glucose-induced osmolarity, sorbitol (a non-digestible sugar) was added to the diet of a control group. Graph shows the average survival \pm s.d. from three independent experiments. Approximately 50 worms were used per condition per each experiment. $P$ values were calculated with respect to control animals in the same experiment using Student's $t$-Test. See Methods section for details. (b) Glucose in the diet protects against chronic exposure to high level of diamide (15 mM). The average curve from three independent experiments is plotted (see also Table 1 and Supplementary Table 2). (c) Transcriptional response to dietary glucose in C. elegans. Pie diagrams demonstrating major functional classes of genes upregulated (left panel) and downregulated (right panel) by glucose diet. L4 stage worms were placed on NGM plates with or without glucose for $\sim 20 \mathrm{~h}$ at $20^{\circ} \mathrm{C}$. Numbers indicate the percentage of genes of the given class. Full list of differentially expressed genes is presented in Supplementary Table 1. (d) Lipids accumulation in response to a high glucose diet. L4 stage wt worms were placed on NGM plates $+/$ - glucose for 3 days at $20^{\circ} \mathrm{C}$ followed by staining with Oil Red O. Scale bar $0.15 \mathrm{~mm}$.

more sensitive to diamide and displayed much weaker glucosemediated protection against oxidants (Fig. $2 \mathrm{~b}$ and Supplementary Fig. 1). These results implicate the enhanced cellular ability to reduce $\mathrm{NADP}^{+}$and GSSG in glucose-mediated protection against oxidative stress and imply that GSH is the major defense system against acute oxidative stress.

Glycogen is an evolutionary conserved antioxidant. Because the de novo synthesis of cytoprotective enzymes is not necessary for glucose-mediated resistance to oxidants (Supplementary Table 1), we expected that the addition of glucose while challenging animals with an oxidant should be sufficient to protect them. Strikingly, however, the same concentration of glucose given to the worms during the oxidant treatment failed to improve survival (Fig. 3a), suggesting that a product of glucose metabolism, and not dietary glucose itself, is protective against oxidants.

In most organisms, excess glucose is promptly stored as glycogen (Fig. 2c). Glycogen synthesis/phosphorolysis is regulated via phosphorylation of cognate enzymes, absent a requirement for gene activation or de novo protein synthesis. Therefore, glucose can be rapidly retrieved from glycogen. RNAi depletion of glycogen synthase $(g s y-1)$ abolished glycogen accumulation, regardless of diet (see below). In the absence of GSY-1, C. elegans become very sensitive to diamide and dietary glucose failed to efficiently protect worms against oxidative stress (Fig. $3 \mathrm{~b}$ and Supplementary Fig. 2). Consistently, glycogen phosphorylase depletion (pyg-1, T22F3.3) increased glycogen accumulation (see below) but failed to protect worms against oxidative stress (Fig. 3b and Supplementary Fig. 2). Thus increased glycogen provides the driving force for the rapid reduction of $\mathrm{NADP}^{+}$and GSSG and detoxification of oxidants in nematodes. Although lipids are also stored on a high glucose diet (Fig. 1d) $9,11,28,29$, their breakdown is much slower than glycogen phosphorolysis and $\mathrm{NADH}$ and $\mathrm{FADH}_{2}$ generated as a result of $\beta$-oxidation cannot support GSSG reduction ${ }^{33}$.

To determine whether the glycogen-dependent protection against oxidative stress also occurs in mammalian cells, we depleted glycogen synthase (GYS-2) in human hepatocytes (Fig. 3c and Supplementary Fig. 3). Liver cells incubated with and without insulin correspond to a high and low glucose dietary state, respectively. Indeed, the high level of glycogen accumulated only in the presence of insulin (Fig. 3d) and that efficiently protected hepatocytes against hydrogen peroxide toxicity (Fig. 3e). Depletion of glycogen synthase prevented glycogen accumulation and sensitized hepatocytes to ROS (Fig. 3d,e). Together, these results demonstrate that the phenomenon of glycogen-mediated protection against oxidative stress is evolutionary conserved from worms to humans.

Glycogen limits C. elegans lifespan on a high glucose diet. Mild oxidative stress delays $C$. elegans aging, supposedly due to the hormesis effect, that is, mobilization of cellular defense systems that would otherwise remain silent ${ }^{19,21}$. Therefore, decreased 
Table 1 | Summary of lifespan experiments with wt C. elegans strains.

\section{C.elegans strain E. coli strain/RNAi Media/treatment}

$\mathrm{N} 2$

N2

N2

$\mathrm{N} 2$

$\mathrm{N} 2$

$\mathrm{N} 2$

$\mathrm{N} 2$

$\mathrm{N} 2$

N2

N2

N2

N2

N2

N2

N2

N2

N2

N2

N2

N2

N2

N2

N2

N2

N2

N2

N2

N2

N2
OP50
OP50
OP50
OP50
OP50
OP50
OP50
OP50
OP50
OP50
OP50
OP50
OP50
OP50
e.v.
e.v.
gsr-1
gsr-1
gspd-
gspd-
e.v.
e.v.
gsy-1
gsy-1
pyg-1
pyg-1
e.v.
gsy-1
pyg-1

NGM

NGM + glucose

$\mathrm{NGM}+15 \mathrm{mM}$ diamide

$\mathrm{NGM}+15 \mathrm{mM}$ diamide + glucose

NGM

NGM + glucose

$\mathrm{NGM}+5 \mathrm{mM}$ diamide

$\mathrm{NGM}+5 \mathrm{mM}$ diamide + glucose

$\mathrm{NGM}+$ paraquat

NGM + paraquat + glucose

NGM

NGM + glucose

NGM + acetamonophen

NGM + acetaminophen + glucose

NGM

NGM + glucose

NGM

NGM + glucose

NGM

NGM + glucose

NGM

NGM + glucose

NGM

NGM + glucose

NGM

NGM + glucose

$\mathrm{NGM}+5 \mathrm{mM}$ diamide + glucose

$\mathrm{NGM}+5 \mathrm{mM}$ diamide + glucose

$\mathrm{NGM}+5 \mathrm{mM}$ diamide + glucose
Mean survival, days \pm s.d. $\quad P$ value

\section{$14.24 \pm 1.23$}

$10.14 \pm 1.03$

$10.33 \pm 0.77$

$12.72 \pm 0.75$

0.00576

Mean increase/decrease, $\% \pm$ s.d.

$13.76 \pm 1.12$

$9.52 \pm 1.00$

$17.19 \pm 0.44$

$18.32 \pm 0.82$

$17.18 \pm 1.87$

$14.04 \pm 0.65$

0.00909

$-28.75 \pm 4.19$

$12.45 \pm 0.50$

$9.33 \pm 0.23$

$12.65 \pm 1.36$

$11.25 \pm 0.09$

0.00029

0.41259

0.00732

$23.62 \pm 12.19$

$15.83 \pm 0.76$

$12.53 \pm 1.24$

$14.03 \pm 0.37$

0.00844

0.01043

0.00044

$0.68 \pm 0.66$

0.03819

0.00123

$9.83+0.54$

$17.14 \pm 1.45$

$12.56 \pm 0.77$

$19.66 \pm 0.3$

$15.41 \pm 0.62$

$18.03 \pm 1.49$

$11.54 \pm 0.27$

0.00422

0.02129

0.06468

0.25163

0.00138

$18.13 \pm 1.45$

$18.41 \pm 0.71$

$15.01 \pm 0.34$
0.38957

0.01113
$-30.82 \pm 3.73$

$25.38 \pm 8.12$

$33.47 \pm 5.55$

$23.63 \pm 0.29$

$2.29 \pm 5.00$

$-24.98 \pm 4.26$

$1.53 \pm 9.28$

$-9.55 \pm 3.43$

$-20.92 \pm 5.29$

$-11.26 \pm 4.87$

$-32.47 \pm 4.97$

$-10.59 \pm 4.08$

$-38.59 \pm 0.38$

$-26.17 \pm 9.85$

$15.23 \pm 10.22$

$-9.78 \pm 7.17$

$5.7 \pm 13.41$

$-32.32 \pm 6.32$

$1.77 \pm 4.61$

$-16.96 \pm 5.04$

The percentage of increase $(+)$ or decrease $(-)$ of the lifespan is shown relative to the control of the same experiment. $P$ values were calculated with respect to control animals in the same experiment using Student's $t$-test (one-tailed distribution and two-sample equal variance). See also Supplementary Table 2 for detail on individual experiments.

lifespan in response to dietary glucose could be explained by the glycogen-driven ability to prevent protein thiol oxidation. This would result in interference with redox-sensitive thiol signalling and downregulation of cytoprotective gene transcription.

To test this hypothesis, we first examined whether lifespan shortening in glucose-fed worms could be reversed by low level of exogenous oxidants. Indeed, diamide eliminated the negative effect of glucose on C. elegans lifespan (Fig. 4a, Table 1). Paraquat also decreased glucose toxicity although its effect was smaller than that of diamide, perhaps because paraquat utilizes some of NADPH, which is required for GSSG reduction, to generate $\operatorname{ROS}^{34}$ (Fig. 4b, Table 1). Furthermore, ubiquitous anti-inflammatory drug acetaminophen (Tylenol) protected worms against glucose toxicity (Fig. 4c). The major side effect of acetaminophen in animals is liver damage caused by severe oxidative stress, which accounts for many life-threatening cases of drug overdose every year ${ }^{35,36}$. Acetaminophen conjugates with cellular GSH, depleting hepatocytes of their primary antioxidant ${ }^{35,36}$. Note that, similar to paraquat or diamide, a very low concentration of acetaminophen also extends C. elegans lifespan on a regular diet ${ }^{37}$. Together our results demonstrate that oxidants and glucose cancel out each other's negative effect on the lifespan (Figs $1 \mathrm{~b}$ and 4 ).

In principle, either low glycogen or elevated ROS could initiate a signal leading to extended lifespan. To discriminate between these two possibilities, we first diminished the reduction of thiols by knocking down GSR ( $g s r-1$ ) or glucose-6-phosphate dehydrogenase ( $g s p d-1)$, the enzyme which generates NADPH, the cofactor required by all GSSG-reducing enzymes (Fig. 2c). GSR or GSPD depletion compromises scavenging of endogenous, as well as exogenously added, ROS without a significant effect on glycogen stores (Supplementary Fig. 4a) ${ }^{32}$. However, both GSR- and GSPD-deficient animals did not live longer than wt and remained susceptible to glucose-mediated life shortening (Fig. 5, Table 1). Thus the low level of reduced GSH, and the resulting elevated ROS, does not increase the lifespan. Instead, the oxidant-mediated depletion of glycogen stores appears to be the primary cause of life extension by oxidants.

To confirm that oxidants do, indeed, deplete glycogen, we monitored the glycogen level in worm cells lysates using an enzymatic assay or in live animals by iodine staining (Fig. $4 \mathrm{~d}$ and Supplementary Fig. 4b). Glucose-fed worms accumulated twofold more glycogen than worms fed a regular diet (Fig. 4d, Supplementary Table 3). Challenging worms with diamide, paraquat or acetaminophen (under the same conditions that resulted in life extension) depleted the glycogen stores (Fig. 4d, Supplementary Fig. 4b and Supplementary Table 3). Thus glycogen is depleted in response to oxidative stress, and notably, the magnitude of lifespan extension by oxidants or shortening by glucose is evidently correlated with changes in glycogen content (Fig. 4 and Supplementary Fig. 4c).

To prove that glycogen diminishes the C. elegans lifespan, we knocked down glycogen synthase (gsy-1) or glycogen phosphorylase (pyg-1, T22F3.3), which breaks down glycogen (Fig. 2c). Irrespective of diet, gsy-1 depletion prevented glycogen accumulation (Fig. 6b,d, Supplementary Table 3) and increased the lifespan of glucose-fed worms (Fig. 6a, Table 1). Curiously, gsy-1 depletion only through the development phase and first 4 days of adulthood was as effective against glucose-mediated 
a

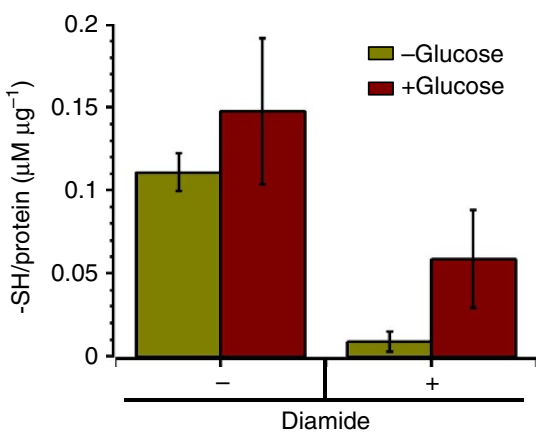

b

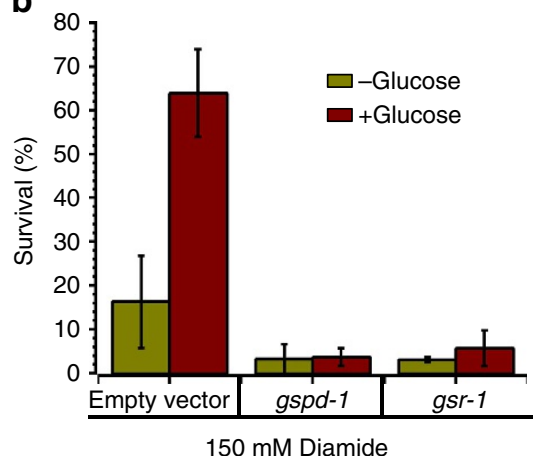

C

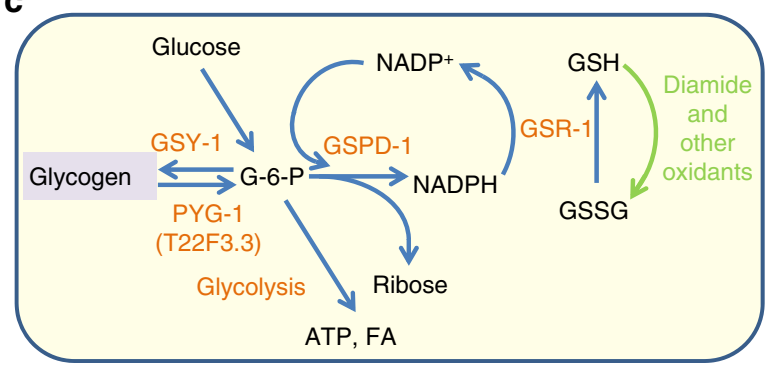

Figure 2 | Glucose-driven glutathione reduction is the major defense against oxidants. (a) Glucose promotes cellular thiols reduction. L4 worms were placed on NGM plates with or without glucose. Two-day-old adults were picked and incubated for 10 min with $(n=400, P=0.035)$ or without $(n=400$, $P=0.13$ ) non-lethal concentration of diamide. Total cellular reduced thiols were quantified in worm lysates by reaction with DTNB (see Methods section) Graph shows the mean \pm s.e. from four independent experiments. (b) Endogenous glutathione is the major defense against oxidants. Glutathione reductase ( $g s r-1)$ or glucose-6-phosphat dehydrogenase ( $g$ spd-1) were knocked down by RNAi, followed by diamide treatment as in Fig. 1a. Graph shows the mean \pm s.d. from at least three independent experiments (e.v.: $n=140, P=0.0003 ;$ gsr-1: $n=120, P=0.129 ; g s p d-1: n=110, P=0.434$ ). $P$ values were calculated with respect to control animals in the same experiment using Student's t-Test. See also Supplementary Fig. 1. (c) Major pathways of glucose utilization and storage. Upon entering the cell, glucose is phosphorylated and is utilized for ATP production and the biosynthesis of macromolecules. Excess glucose is stored as glycogen or converted to fatty acids (FA). A large portion of glucose is used for NADP ${ }^{+}$reduction, which is required for subsequent reduction of oxidized glutathione and thioredoxin. Glucose thus acts as a powerful (albeit indirect) antioxidant. GSY-1, glycogen synthase; PYG-1(T22F3.3), glycogen phosphorylase; GSPD-1, glucose-6-phosphate dehydrogenase; GSR-1, glutathione reductase.

toxicity as its life-long depletion (Supplementary Fig. 5). This result indicates that glycogen accumulation in early age is more detrimental to animal health.

As expected, the deficiency of glycogen phosphorylase increased the amount of stored glycogen resulting in even shorter lifespan on a high glucose diet (Fig. 6a,b, Table 1). However, on glucose-free media, pyg-1-deficient worms, while still accumulating glycogen, did not live shorter (Fig. 6a,b), indicating that glycogen accelerates aging only on a high glucose diet.

To demonstrate that the oxidants are not capable of reversing the negative effect of glucose on lifespan, if the glycogen level remains high, we fed wt, gsy-1 and pyg-1 worms both glucose and diamide. As expected, diamide depleted glycogen in wt and $g s y$-1 animals (Fig. 6b,d) and increased their lifespan (Fig. 6c and Table 1). In contrast, diamide failed to increase the lifespan of pyg-1-deficient animals, which accumulated the high level of glycogen (Fig. 6b,c).

Together these results demonstrate that it is not the antioxidant capacity (high GSH/GSSG ratio) or low ROS but rather endogenous glycogen that signals to reduce the lifespan of C. elegans fed a high glucose diet. Diamide and other oxidants deplete glycogen via the PPP/GSH pathway, thereby switching off this life-shortening signal (Fig. 2c).

Glycogen interferes with the daf-2 antiaging signalling. Inhibition of IIS substantially extends the C. elegans lifespan ${ }^{38}$. Mutations in the insulin/insulin-like growth factor receptor gene, daf-2, induce daf-16-mediated transcription of many cytoprotective and antiaging genes ${ }^{39-41}$. Dietary glucose is thought to activate $D A F-2$ to repress daf-16-regulated transcription ${ }^{13}$. DAF-16 enters the nucleus in glucose-fed daf-2 worms, but the antiaging properties of these animals are somehow subjugated by glucose ${ }^{13}$.

Worms, exposed to dietary glucose postdevelopmentally, increased glycogen accumulation by $\sim 30 \%$ and live $\sim 5$ days shorter than daf- 2 control animals fed a regular diet (Fig. 7a,b, Supplementary Fig. 6, Supplementary Table 3). To determine whether glycogen initiates a signal that decreased longevity of glucose fed daf-2 worms, we used RNAi to deplete gsy-1 (Fig. 7b and Supplementary Fig. 6). Although gsy-1 daf-2 animals, which do not make glycogen, live $\sim 5 \%$ shorter compared to daf-2 worms, their lifespan was not further affected by glucose (Fig. $7 \mathrm{a}$ and Table 2). Consistently, pyg-1 RNAi further increase glycogen level in daf-2 worms and dramatically shorten their lifespan on a high glucose diet (Fig. 7a and Table 2), as if more glycogen is required to counterbalance low IIS.

Note that on a glucose-free diet the glycogen level is higher in both daf-2 and pyg-1 daf-2 worms compared to wt (Figs $6 \mathrm{~b}$ and $7 \mathrm{~b}$ ). However, those animals do not live shorter. These results demonstrate again that glucose alone (such as in the case of $g s y-1$ worms), or glycogen alone, do not shorten the lifespan. Glycogen accumulated in the presence of high glucose interferes with low IIS, resulting in a shortened lifespan of daf-2 animals.

Glycogen regulates SOD-3 and aging independently of DAF-16. FOXO/DAF-16 transcription factor contributes greatly to C. elegans longevity ${ }^{41}$. Expression of sod-3 in intestinal cells is a 

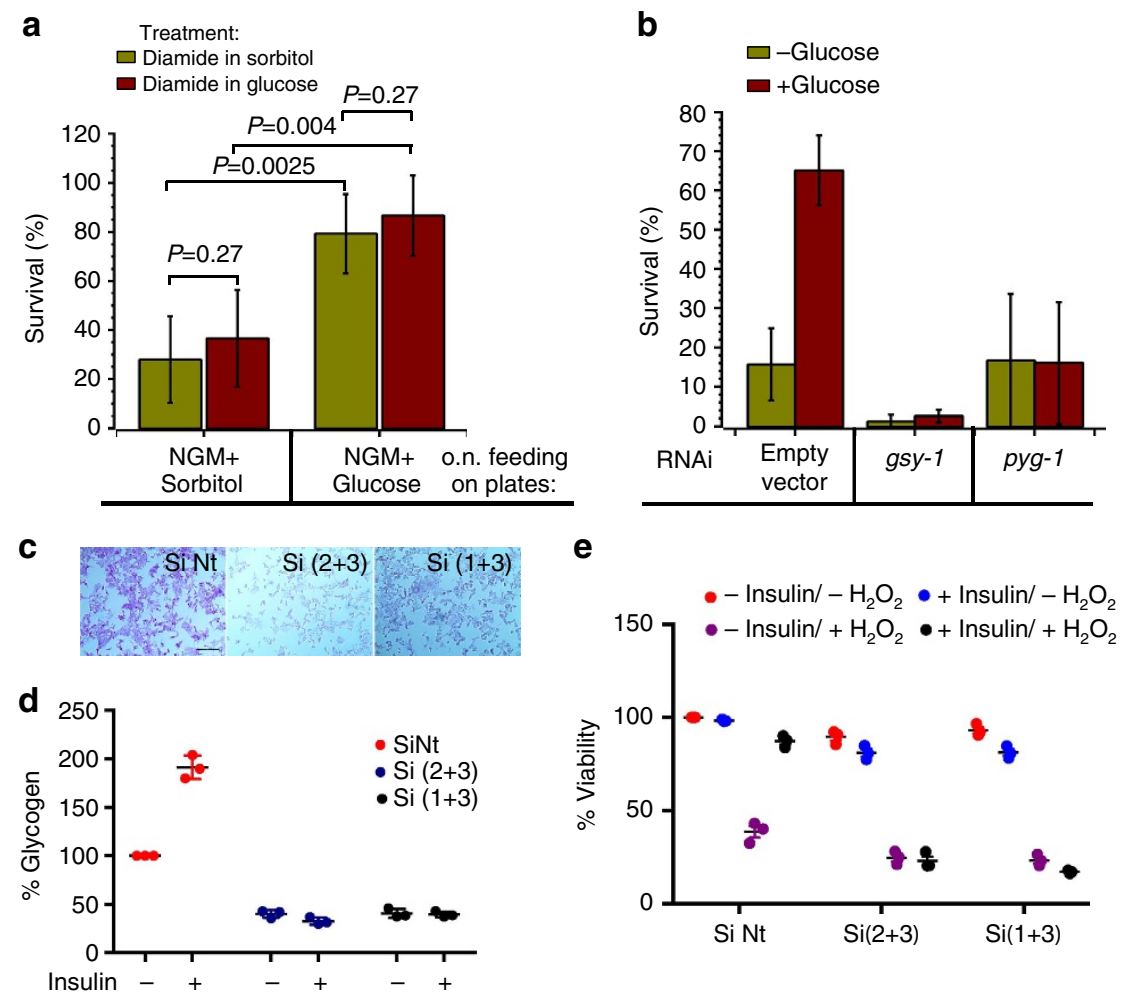

Figure 3 | Glycogen protects C. elegans and hepatocytes against oxidative stress. All graphs show the average survival \pm s.d. from at least three independent experiments. Approximately 50 worms were used per condition per each experiment. (a) Stored glucose metabolite(s), not glucose in the media, protects C. elegans from oxidants. L4 stage animals grown on NGM plates + sorbitol or + glucose for $20 \mathrm{~h}$ were picked and transferred to M9 containing sorbitol and diamide (green bars) or glucose and diamide (red bars). (b) Glycogen storage is required for C. elegans protection against oxidants. N2 worms fed on NGM plates seeded with E. coli HT115 harbouring either empty plasmid vector or plasmid vectors expressing gsy-1 or pyg-1 RNAi. L4 stage animals were transferred to NGM plates with sorbitol (green bars) or with glucose (red bars) for $20 \mathrm{~h}$ and then treated with $150 \mathrm{mM}$ diamide (e.v.: $n=130$, $P=0.00001 ;$ gsy-1: $n=155, P=0.105 ;$ pyg-1: $n=180, P=0.479)$. $P$ values were calculated with respect to control animals in the same experiment using Student's t-Test. See also Supplementary Fig. 2. (c) PAS staining of HepG2 cells after glycogen synthase 2 knockdown by siRNA. SiNt, non target siRNA; $\mathrm{Si}(2+3)$, combination of siRNA GYS2.2 and GYS2.3; Si(1+3), combination of siRNA GYS2.1 and GYS2.3. Scale bar $100 \mu \mathrm{m}$. (d) Relative glycogen content in HepG2 cells treated with insulin and GYS-2 siRNA. Error bars represent s.d. of three independent experiments. (e) Glycogen protects hepatocytes against peroxide. HepG2 cell were depleted of glycogen synthase 2 by siRNAi and then incubated with $50 \mu \mathrm{M} \mathrm{H} \mathrm{H}_{2}$ for 30 min. Cell viability was assayed after $24 \mathrm{~h}$. Insulin was added to a final concentration of $25 \mu \mathrm{M}$. Error bars represent s.d. of three independent experiments.

reliable marker of DAF-16 antiaging activity ${ }^{42}$. In accordance with previously published data ${ }^{13}$, we found that a high glucose diet decreased sod-3 expression by $\sim 50 \%$ in wt and by $\sim 30 \%$ in daf-2 worms (Supplementary Fig. 7 and Supplementary Table 4). Depletion of glycogen synthase $(g s y-1)$ increased sod-3 expression $\sim 2.5$ times in wt and $\sim 1.5$ times in daf- 2 worms (Supplementary Fig. 7). Remarkably, glucose fails to inhibit sod-3::GFP accumulation in glycogen-depleted worms (Supplementary Fig. 7). We noticed that effect of daf-2 mutation on sod-3 expression is much stronger than from glycogen depletion and results in GFP accumulation mainly in the head area (compare Supplementary Fig. 7a and 7c). In contrast, glycogen depletion mostly increases fluorescence in the tail area (Supplementary Fig. 7). To clarify this, we subjected wt worms to gsy-1 and daf-16 double RNAi treatment. Surprisingly, the depletion of DAF-16 increased the tail accumulation of GFP in wt worms and had no effect on sod-3 expression in gsy-1-deficient worms (Supplementary Fig. 7e). Together these results indicate that the tail expression of sod-3 is not a marker of DAF-16-mediated transcription.

To further address the role of daf-16 in glycogen-mediated life shortening, we depleted $g s y-1$ in daf-16 and daf- 2 daf-16 worms. In our experimental set-up (adults only glucose treatment), glucose decreased the lifespan of $d a f-16$ and, to a lesser extent, of daf-2 daf-16 animals (Fig. 8a,b, Supplementary Table 2). Remarkably, gsy-1 RNAi increased the lifespan of both these strains irrespective of their diet. These results indicate that glycogen shortens the lifespan independently of daf-16 (Fig. 8 and Table 2).

AMPK is required for high glucose tolerance in $g s y$ - 1 worms. Glycogen dramatically shortens the lifespan of daf-2 animals on a high glucose diet (Fig. 7a), implying that it must interfere with a certain DAF-2-regulated antiaging pathway. DAF-16 and AMPK are both required for the full lifespan extension of daf-2 animals ${ }^{43,44}$. Moreover, the deletion of $a a k-2$, an AMPK ortholog in C. elegans, shortens the lifespan of daf-2 and daf-16 (refs 43,45,46). Paraquat treatment does not extend aak-2 lifespan ${ }^{47}$. Finally, in human cells, glycogen directly binds and sequesters AMPK to downregulate its activity ${ }^{48}$. Together, these observations suggest that AMPK is a target of glycogen-induced regulation of $C$. elegans lifespan. To address this possibility, we depleted gsy-1 in aak-2 worms and compared their lifespan on regular and high glucose diet. In agreement with the published data, glucose further shortened the lifespan of aak-2 (Fig. 8c) ${ }^{13}$. However, gsy-1 depletion did not extend its lifespan on a high glucose diet (Fig. 8c and Table 2), indicating that AMPK is indeed required for life extension in the absence of glycogen. Thus 

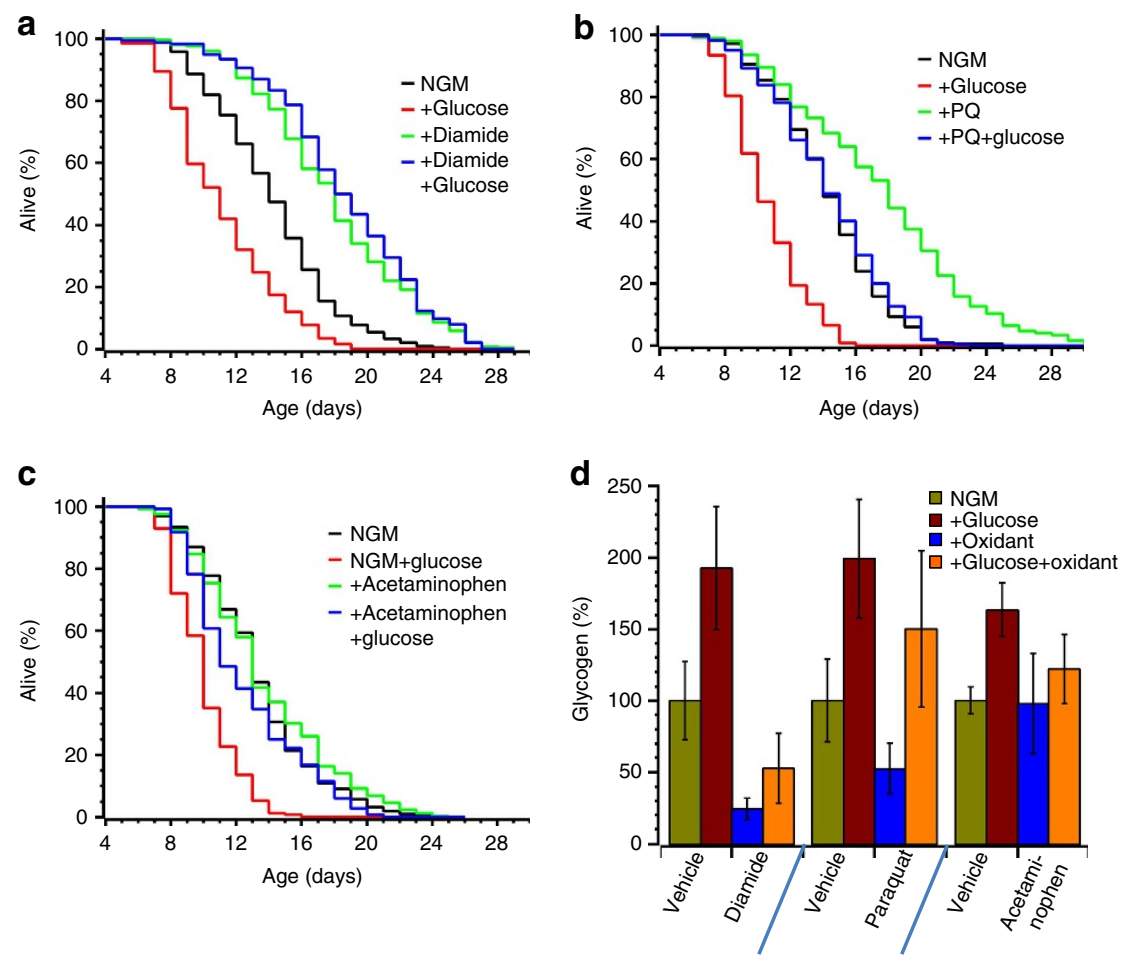

Figure 4 | Glycogen depletion by oxidants delays aging. (a-c) The short lifespan of glucose-fed C. elegans is reversed by $5 \mathrm{mM}$ diamide (a), $0.2 \mathrm{mM}$ paraquat (b) and $5 \mathrm{mM}$ acetaminophen (c). Half of L4 staged animals were placed on NGM plates $+/-$ glucose (red and black curves), the other half was transferred to NGM plates + / - glucose supplemented with diamide (a), paraquat (b) or acetaminophen (c). For each graph, the average curve from three independent experiments is plotted (see also Table 1 and Supplementary Table 2). (d) Glycogen content is increased in worms fed a high glucose diet and is depleted by oxidants. Worms were grown and fed as in panel a-c. Glycogen content of 3-day-old animals was determined by the enzymatic assay and normalized to total protein. The graph shows changes in glycogen levels from at least three independent experiments (\% \pm s.d.) between the experimental and control groups of animals (see also Supplementary Table 3).
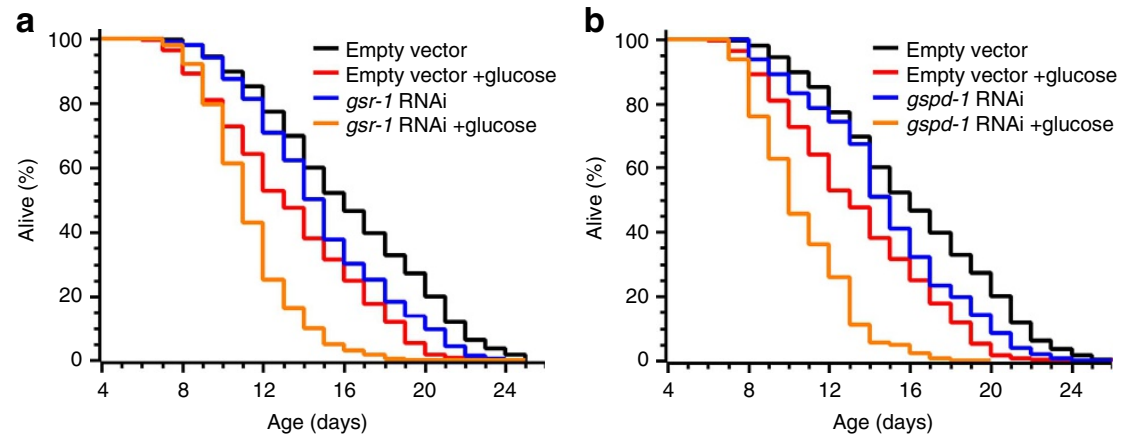

Figure 5 | Blocking the endogenous antioxidant system does not influence glucose-mediated life shortening. (a and b) Wt worms grew on NGM plates seeded with E. coli HT115 harbouring either an empty vector (black and red curves) or a plasmid expressing either double-stranded gsr-1 (a) or gspd-1 (b) RNAi (blue and orange curves). L4 stage animals were transferred to NGM plates without (black and blue curves) or with (red and orange curves) glucose (see also Supplementary Table 2). For RNAi efficiency, see Fig. $2 b$.

the lifespan of C. elegans is determined by a combination of daf-2- and glycogen-to-AMPK-mediated signalling (Fig. 8d).

\section{Discussion}

Glucose is essential for animal life. Yet, its excess has been linked to a multitude of pathological conditions ${ }^{2,3}$ and diminished lifespan across evolutionary genera ${ }^{4,5,13}$. Several attempts have been made to explain glucose toxicity, and the IIS pathway was proposed to be the major target ${ }^{13}$. However, genetic inactivation of IIS in worms does not mitigate the negative effect of glucose on lifespan ${ }^{13}$ (Fig. 7a), indicating that other pathways must be involved. Here we show that glycogen signalling plays the principal role in limiting the lifespan of nematodes fed a high glucose diet (Fig. 8d).

Our results indicate that glycogen and IIS are the two distinct signalling pathways controlling the lifespan in nematodes (Fig. 8d). On a high glucose diet, IIS is activated to suppress DAF-16-dependent transcription. Simultaneously, accumulation of glycogen inhibits AMPK activity. Together this leads to a dramatic shortening of lifespan (Fig. 6a). Inhibition of glycogen synthesis by gsy-1 RNAi relieves AMPK inhibition and, 
a

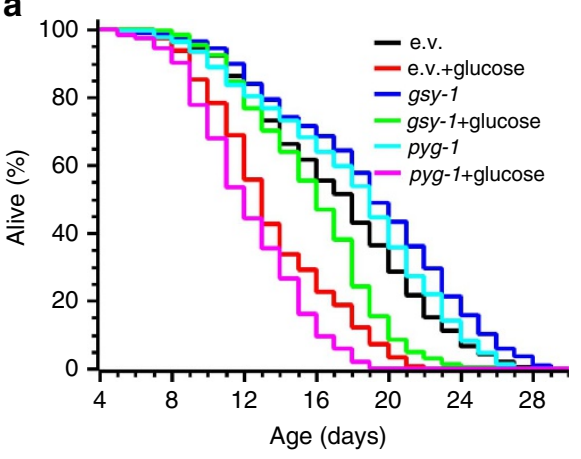

C

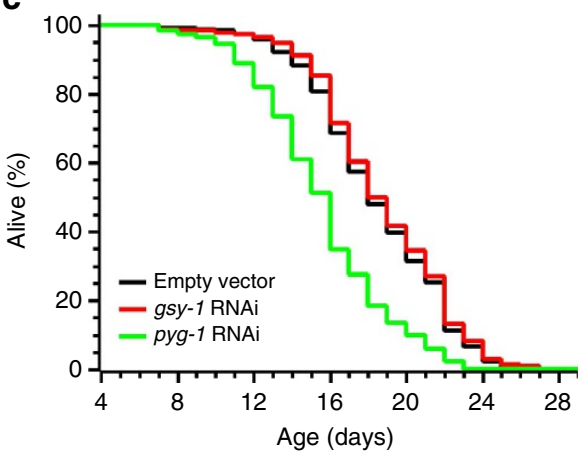

b

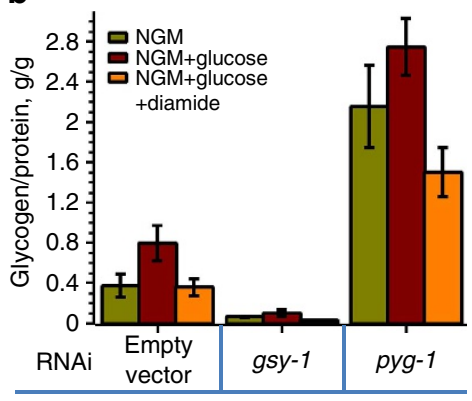

d

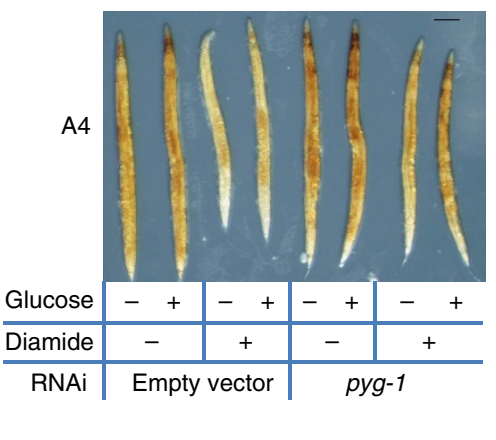

Figure 6 | Glycogen accumulation is responsible for glucose-mediated life shortening. (a) Worms lacking glycogen deposition live much longer on a high glucose diet. N2 worms were fed on NGM plates seeded with E. coli HT115 harbouring either empty vector (black and red curves) or plasmids expressing either gsy-1 (blue and green curves) or pyg-1 (cyan and magenta curves) RNAi. L4 stage animals were transferred to NGM plates without (black, blue and cyan curves) or with (red, green and magenta curves) glucose. The graph shows the average of three independent experiments (see also Supplementary Table 2). (b) Glycogen content in gsy-1 and pyg-1 knockdown worms. Animals were grown on NGM (green bars), NGM $+2 \%$ glucose (red bars) or $\mathrm{NGM}+2 \%$ glucose $+5 \mathrm{mM}$ diamide (orange bars) agar plates as in panel a or c. Seven-day-old worms were then picked, washed and the amount of glycogen determined and normalized to the total protein. The graph shows the average \pm s.d. of at least three independent experiments (see also Supplementary Table 3). (c) Diamide fails to extend the short lifespan of glycogen phosphorylase-deficient worms fed on high glucose diet. N2 worms were fed on NGM plates seeded with E. coli HT115 harbouring either empty vector (black curve) or plasmid vector expressing either gsy-1 (red curve) or pyg-1 (green curve) RNAi. L4 stage animals were transferred to NGM plates with $2 \%$ glucose and $5 \mathrm{mM}$ diamide. The graph shows the average of three independent experiments (see also Supplementary Table 2). (d) Diamide does not efficiently deplete glycogen in pyg-1-deficient worms. A representative image of four-day-old worms stained for glycogen with iodine is shown. Scale bar $0.15 \mathrm{~mm}$. Experimental conditions were as in panel $\mathbf{c}$.
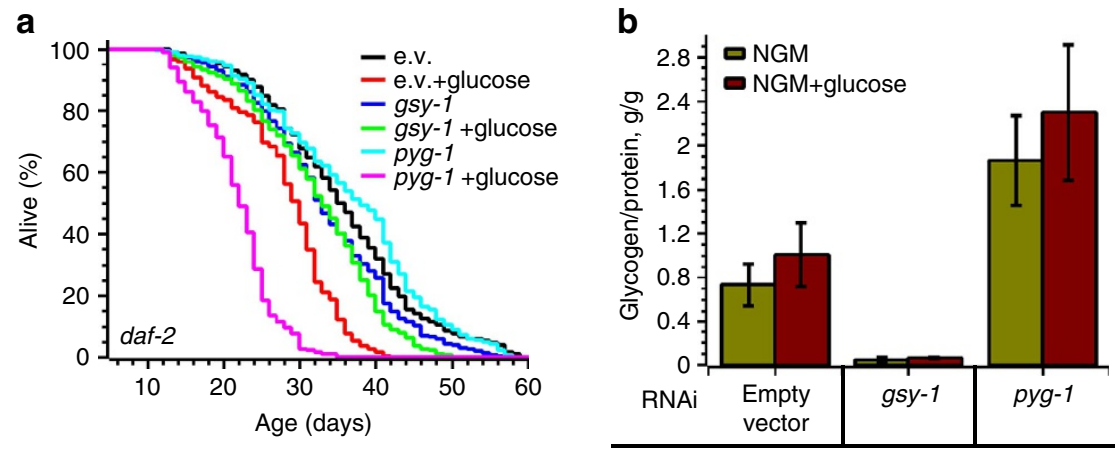

Figure 7 | Glycogen is responsible for glucose-mediated life shortening of daf-2 worms. (a) Inverse correlation between glycogen production and lifespan of daf-2 (e1370, hypomorph) worms fed a high glucose diet. Worms were grown on NGM plates seeded with E. coli HT115 harbouring either empty vector (black and red curves) or plasmids expressing either gsy-1 (blue and green curves) or pyg-1 (cyan and magenta curves) RNAi. L4 stage animals were then transferred on NGM plates without (black, blue and cyan curves) or with (red, green and magenta curves) glucose. The graph shows the average of three independent experiments (see also Supplementary Table 2). (b) Glycogen content in daf-2 worms depleted of gsy-1 or pyg-1. Worms were grown as in panel a on NGM (green bars) or NGM $+2 \%$ glucose (red bars) plates. Seven-day-old worms were picked, washed and the amount of glycogen determined and normalized to the total protein. The graph shows the average \pm s.d. of at least three independent experiments (see also Supplementary Table 3 ).

consistently, increases the lifespan of wt, daf-2 and daf-16 worms fed a high glucose diet (Figs 6a,7a and 8). The latter indicates that glycogen and $d a f-16$ are parallel signalling pathways independently controlling the lifespan. In GSY-1-deficient worms fed glucose, DAF-2 signalling could still suppress DAF-16; however, this effect is countered by low glycogen, which relieves the AMPK inhibition (Fig. 8d), thereby promoting daf-16 activation $^{49}$. 
Table 2 | Summary of lifespan experiments with mutant C. elegans strains.

\begin{tabular}{|c|c|c|c|c|c|}
\hline C.elegans strain & E. coli strain/RNAi & Media/treatment & Mean survival, days \pm s.d. & $P$ value & Mean increase/decrease, $\% \pm$ s.d. \\
\hline $\begin{array}{l}\text { daf-2 } \\
\text { daf-2 } \\
\text { daf-2 } \\
\text { daf-2 } \\
\text { daf-2 } \\
\text { daf-2 }\end{array}$ & $\begin{array}{l}\text { e.v. } \\
\text { e.v. } \\
\text { gsy-1 } \\
\text { gsy-1 } \\
\text { pyg-1 } \\
\text { pyg-1 }\end{array}$ & $\begin{array}{l}\text { NGM } \\
\text { NGM + glucose } \\
\text { NGM } \\
\text { NGM + glucose } \\
\text { NGM } \\
\text { NGM + glucose }\end{array}$ & $\begin{array}{c}34.83 \pm 0.35 \\
29.29 \pm 0.46 \\
32.86 \pm 0.73 \\
32.95 \pm 1.31 \\
37.34 \pm 3.86 \\
21.66 \pm 1.03\end{array}$ & $\begin{array}{c}0.00004 \\
0.00678 \\
0.03744 \\
0.16279 \\
0.00002\end{array}$ & $\begin{array}{c}-15.91 \pm 1.16 \\
-5.66 \pm 2.22 \\
-5.41 \pm 3.09 \\
7.15 \pm 10.43 \\
-37.83 \pm 2.37\end{array}$ \\
\hline $\begin{array}{l}\text { daf }-16 \\
\text { daf-16 } \\
\text { daf- } 16 \\
\text { daf-16 }\end{array}$ & $\begin{array}{l}\text { e.v. } \\
\text { e.v. } \\
\text { gsy-1 } \\
\text { gsy-1 }\end{array}$ & $\begin{array}{l}\text { NGM } \\
\text { NGM + glucose } \\
\text { NGM } \\
\text { NGM + glucose }\end{array}$ & $\begin{array}{c}12.35 \pm 1.2 \\
9.55 \pm 0.49 \\
13.95 \pm 0.88 \\
10.79 \pm 0.23\end{array}$ & $\begin{array}{l}0.01001 \\
0.06641 \\
0.04679\end{array}$ & $\begin{array}{l}-22.21 \pm 8.2 \\
-13.35 \pm 8.3 \\
-12.17 \pm 8.16\end{array}$ \\
\hline $\begin{array}{l}\text { daf-2; daf-16 } \\
\text { daf-2; daf-16 } \\
\text { daf-2; daf-16 } \\
\text { daf-2; daf-16 }\end{array}$ & $\begin{array}{l}\text { e.v. } \\
\text { e.v. } \\
\text { gsy-1 } \\
\text { gsy-1 }\end{array}$ & $\begin{array}{l}\text { NGM } \\
\text { NGM + glucose } \\
\text { NGM } \\
\text { NGM + glucose }\end{array}$ & $\begin{array}{l}13.15 \pm 1.24 \\
11.53 \pm 0.72 \\
14.56 \pm 1.16 \\
12.28 \pm 0.19\end{array}$ & $\begin{array}{l}0.03867 \\
0.11200 \\
0.10455\end{array}$ & $\begin{array}{c}-12.24 \pm 0.26 \\
11.02 \pm 8.25 \\
-6.18 \pm 8.50\end{array}$ \\
\hline $\begin{array}{l}\text { aak-2 } \\
\text { aak-2 } \\
\text { aak-2 } \\
\text { aak-2 }\end{array}$ & $\begin{array}{l}\text { e.v. } \\
\text { e.v. } \\
\text { gsy-1 } \\
\text { gsy-1 }\end{array}$ & $\begin{array}{l}\text { NGM } \\
\text { NGM + glucose } \\
\text { NGM } \\
\text { NGM + glucose }\end{array}$ & $\begin{array}{c}12.76 \pm 0.16 \\
10.23 \pm 0.55 \\
12.54 \pm 0.77 \\
9.89 \pm 0.32\end{array}$ & $\begin{array}{l}0.00076 \\
0.32458 \\
0.00008\end{array}$ & $\begin{array}{l}-19.85 \pm 3.35 \\
-1.79 \pm 4.87 \\
-22.53 \pm 2.5\end{array}$ \\
\hline
\end{tabular}

The percentage of increase $(+)$ or decrease $(-)$ of the lifespan is shown relative to the control of the same experiment. $P$ values were calculated with respect to control animals in the same experiment using Student's $t$-test (one-tailed distribution and two-sample equal variance). See also Supplementary Table 2 for detail on individual experiments.

a

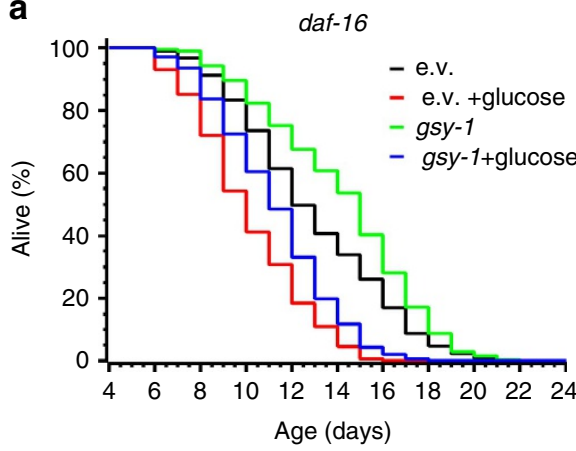

C

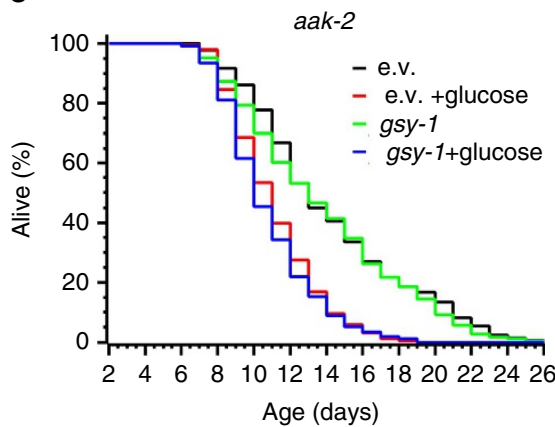

b

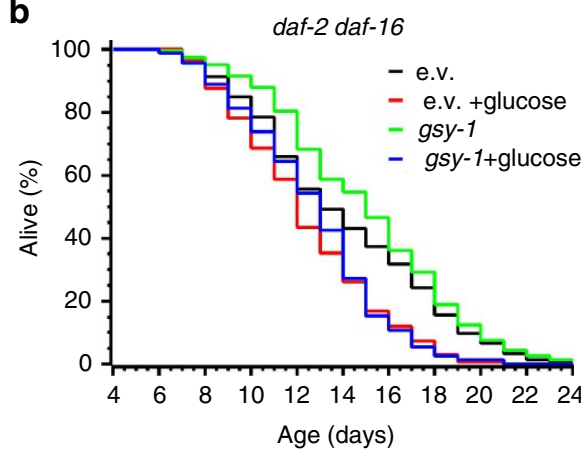

d

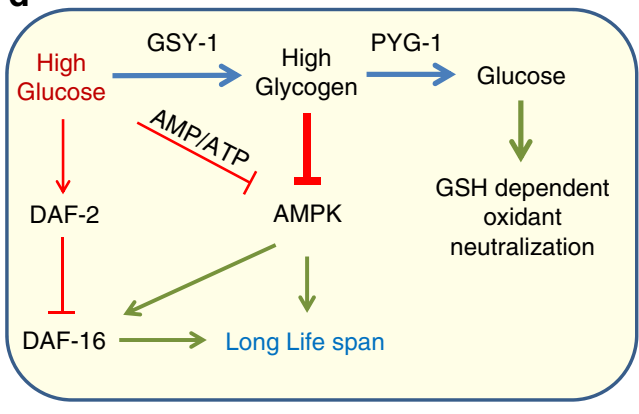

Figure 8 | AMPK is required for life extension in glycogen-depleted C. elegans. (a-c) Depletion of glycogen extends the lifespan of daf-16 (a) and daf-2 daf-16 (b) worms but fails to increase the longevity of aak-2 animals (c). Worms were fed on NGM plates seeded with E. coli HT115 harbouring either empty vector (black and red curves) or plasmids expressing gsy-1 (blue and green curves) RNAi. L4 animals were transferred to NGM plates without (black and green curves) or with (red and blue curves) glucose. The graph shows the average of three independent experiments (see also Table 2 and Supplementary Table 2). (d) Interplay between glycogen and insulin-like signalling controls C. elegans lifespan. A high glucose diet simultaneously activates insulin-like signalling and induces glycogen storage. IIS suppression of DAF-16-mediated transcription and inhibition of AMPK by glycogen shorten the lifespan. Pro-aging glycogen signalling overcomes the antiaging effect of IIS inhibition (daf-2). Inactivation of glycogen synthesis (gsy-1) prevents glycogen accumulation, relieves AMPK and extends the lifespan in wt, daf-16 and daf-2 worms on a high glucose diet. Oxidants force the glucose flow through PPP to regenerate cellular GSH, thus depleting glycogen stores and extending the lifespan.

Activation IIS by glucose explains why $g s y-1$ worms on high glucose live slightly shorter comparing to wt on a regular diet (Fig. 6a). Conversely, in daf-2 background, where the negative effect of glucose on lifespan via the IIS pathway is abrogated, gsy-1 depletion completely restored the lifespan (Fig. 7a). Although inconsistent with published data ${ }^{13,27}$, the negative effect of glucose on the lifespan of daf-16 worms, which we observe here, fits our model. Glycogen accumulated in daf-16 worms shortens their lifespan via the AMPK pathway, while activation of IIS signalling by glucose decreases the lifespan of AMPK-deficient worms (Fig. 8).

Glycogen phosphorylase-deficient worms accumulate excess of glycogen and, in accordance with our model, live shorter on a 
high glucose diet, irrespective of IIS (Fig. 8d). However, on a glucose-free diet accumulation of glycogen does not compromise their lifespan (Figs 6a and 7a). This clearly demonstrates that both dietary glucose and glycogen accumulation are required for shortening the lifespan. AMPK is primarily regulated by AMP and $\mathrm{ATP}^{43,50}$ and its activity is oppositely controlled by AMP and glycogen in mammals ${ }^{48}$. Consistently, we found that the level of ATP is lower in pyg-1-deficient worms compared to wt (Supplementary Fig. 8), apparently, because most of cellular glucose is trapped in glycogen on sugar-free diet. Low ATP should oppose glycogen-dependent inhibition of AMPK (Fig. 8d).

Although the molecular details of glycogen-mediated regulation of AMPK remain to be characterized, it is reasonable to propose that the pharmacological inhibition of this pathway could be beneficial. Liver glycogen helps to maintain the steady level of circulating glucose between meals. However, the excess glycogen observed in many disorders ${ }^{51,52}$, including several types of glycogen-storage diseases and diabetes may be a root cause of concomitant pathologies. Patients with type I, VI and IX glycogen storage diseases accumulate high levels of glycogen and are at increased risk of developing cirrhosis, hepatocellular adenomas and carcinomas ${ }^{51,52}$. The role of glycogen in development of these pathologies, however, has never been directly addressed. Chronic inhibition of glycogen phosphorylase in rats causes liver inflammation and damage, supporting the link between glycogen excess and disease ${ }^{53}$. In contrast, adults with liver glycogen synthase deficiency are typically asymptomatic and rely on gluconeogenesis to meet their glucose needs ${ }^{54,55}$. Thus it is possible that liver glycogen is expendable during adulthood, and excess glycogen resulting from a high carbohydrate diet may actually be harmful.

Our experiments with C. elegans support this idea. Gsy-1 animals produce very little glycogen. They manifest no abnormalities in adulthood, live $\sim 15 \%$ longer than wt animals and are resistant to a high glucose diet (Figs 6a and 7a). In contrast, worms that accumulate glycogen when fed a high sugar diet have a shortened lifespan, irrespective of their level of insulin signalling (Figs $6 \mathrm{a}$ and $7 \mathrm{a}$ ). Thus one of the important outcomes of this study is that dietary glucose is not harmful unless it can be converted to glycogen.

Our findings offer the enticing possibility that the harm imposed by dietary glucose in adults could be reduced by suppressing liver glycogen accumulation with glycogen synthase inhibitors, or, alternatively, by promoting glycogen depletion with oxidants (Figs 2c and 4). A growing body of evidence associates mild oxidative stress, for example, from physical exercise ${ }^{56}$, with longer healthspan, while antioxidants have the opposite effect $^{22}$. Our results indicate that glucose can be viewed as a 'super' antioxidant. Large glycogen depots allow much faster reduction of GSSG and therefore rapid ROS scavenging upon sudden oxidative insult (Figs 1a, 2a and 3a,e). Hence, by applying small amount of oxidants one can drain glycogen stores through PPP metabolism, relieve AMPK inhibition and restore normal lifespan of animals on a high glucose diet (Fig. 8). Considering the high evolutionary conservation of glycogen metabolism, we propose that limited amounts of a therapeutically accepted oxidant could, in principle, be used to lower hepatic glycogen stores.

\section{Methods}

Strains and growth conditions. Wt C. elegans (N2), CF1038 (daf-16(mu86) I), TG38 (aak-2(gt33)), CF1553 (muIs84 ((pAD76) sod-3p::GFP + rol-6)), CF1580 (daf-2(e1370) III; muIs84 ((pAD76) sod-3p::GFP + rol-6)), HT1890 (daf16(mgDf50) I; daf-2(e1370) III) and CB1370 (daf-2(e1370) III) strains were obtained from the Caenorhaditis Genetics Center. The worms were handled according to standard methods ${ }^{57}$. Strains were grown on nematode growth media (NGM) agar plates at $20^{\circ} \mathrm{C}$ except for the daf-2 strain, which was grown at $15^{\circ} \mathrm{C}$ until L4 stage. E. coli OP50 bacteria were grown overnight in LB and $50 \mu$ l were spread on top of the agar plates. Plates were incubated for $\sim 20 \mathrm{~h}$ at $25^{\circ} \mathrm{C}$ and then for at least $1 \mathrm{~h}$ at $20^{\circ} \mathrm{C}$ before worms were transferred onto them.

For RNAi experiments, eggs were isolated by treating adult hermaphrodites with alkaline hypochlorite and allowed to develop and grow for two generation on specific RNAi-expressing bacterial strains before being used or lifespan and stressresistance analysis. First generation was used only in case worms fed with bacteria expressing gspd-1 RNAi, as the second generation does not develop. E. coli HT115 strains harbouring plasmid expressing double-strand RNAi against C.elegans gsy-1, pyg-1, gsr-1 and gspd-1 genes were purchased from the Thermo Scientific collection and single-colony isolates were purified and sequenced to prove the presence of the correct insert. Overnight cultures of E. coli HT115 bacteria harbouring RNAi-expressing plasmid or empty plasmid vector control (pL4440) were grown in LB with $100 \mathrm{~m} \mathrm{~kg} \mathrm{ml}^{-1}$ of carbenicillin, concentrated 4 times and $50 \mu \mathrm{l}$ were spread atop NGM agar plates supplemented with $100 \mu \mathrm{g} \mathrm{ml}^{-1}$ carbenicillin and $1 \mathrm{mM}$ IPTG. Seeded plates were incubated for at least $1 \mathrm{~h}$ at $20^{\circ} \mathrm{C}$ before worms were transferred onto them.

Lifespan analysis. Lifespans were monitored at $20^{\circ} \mathrm{C}$ as described previously ${ }^{58,59}$ All experiments were repeated at least three times and $\sim 100$ worms were used for each experiment. In all cases, stage L 4 worms were used at $t=0$ for lifespan analyses. Worms were judged to be dead when they ceased pharyngeal pumping and did not respond to prodding with a platinum wire. Worms with internal hatching were removed from the plates and not included in lifespan calculations. Data were analysed and Boltzmann sigmoid survival curves generated using the SciDAVis statistical analysis software package. Mean lifespans were compared in Microsoft Excel using the Student's $t$-test, assuming one-tailed distribution and two-sample equal variance. All lifespan plots represent the composites of all independent experiments tabulated in Supplementary Table 2.

In all experiments, worms were allowed to develop until stage L4 on NGM plates at $20^{\circ} \mathrm{C}$ (with the exception of daf-2, which developed at $15^{\circ} \mathrm{C}$ ) and then transferred to the plates with the indicated additives. Where indicated, glucose ( $2 \%$ final concentration) was mixed in NGM plates. Oxidants were evenly distributed on wet NGM (or NGM with glucose) plates to reach to a final concentration of: $5 \mathrm{mM}$ diamide, $0.2 \mathrm{mM}$ paraquat or $5 \mathrm{mM}$ acetaminophen. Diamide and paraquate stock solutions were prepared in water, acetaminophen was dissolved in ethanol and the same concentration of the solvent was used in control plates. Plates were dried for $30 \mathrm{~min}$ before seeding with bacteria. As all oxidants have some bacteriostatic effect, the overnight bacterial cultures were concentrated 8 times and $50 \mu \mathrm{l}$ were spread atop agar plates.

Stress-resistance assays. Resistance to diamide or paraquat was assessed essentially as described ${ }^{60}$. Worms were allowed to develop and grow on agar plates at $20^{\circ} \mathrm{C}$ until they reached stage L4 and then transferred to NGM $+50 \mu \mathrm{g} \mathrm{ml}^{-1}$ kanamycin plates with $2 \%$ sorbitol or glucose and incubated for $\sim 20 \mathrm{~h}$ at $20^{\circ} \mathrm{C}$. It was important to add sorbitol in control to match the osmolarity of high glucose diet. Kanamycin prevents sorbitol and glucose consumption by bacteria. Worms (40-80 animals per each experimental condition) were collected into microcentrifuge tubes with $0.25 \mathrm{ml}$ of $\mathrm{M} 9$ buffer with sorbitol or glucose supplemented with oxidant. The final concentration of diamide was 125 or $150 \mathrm{mM}$, and paraquat $-125 \mathrm{mM}$. Tubes were incubated at $20^{\circ} \mathrm{C}$ with agitation for 1-2 h. To remove the oxidant, worms were washed in M9 buffer three times and then transferred to NGM plates seeded with bacteria. Plates were incubated for $48 \mathrm{~h}$ at $20^{\circ} \mathrm{C}$ and then live worms were counted and the survival rate was calculated as a ratio of those alive to the total number of worms transferred to the plate after oxidant treatment. All experiments were repeated at least three times and the average \pm s.d. presented in the Figs.

Determination of glycogen content in C. elegans. Worms were allowed to develop and grow on agar plates at $20^{\circ} \mathrm{C}$ until they reached stage $\mathrm{L} 4$ and then transferred to NGM plates with or without glucose. At certain ages, worms were collected in micro-centrifugal tubes, washed quickly two times with distilled water, most of the liquid was removed and the worms were flash frozen in liquid nitrogen and stored at $-80^{\circ} \mathrm{C}$ until further analysed. For analysis, worms were resuspended in $\sim 70 \mu \mathrm{l}$ of $\mathrm{dH}_{2} \mathrm{O}$ and immediately boiled for $5 \mathrm{~min}$ to inhibit enzymatic degradation of glycogen. Tubes were chilled on ice and the worms were grinded with a disposable pestle and aggregated protein and other insoluble material separated by centrifugation. A Glycogen Assay Kit (Sigma, MAK016-1KT) was used to determine glycogen content in the supernatant. As most of the proteins precipitate after boiling, the pellets were dissolved in $\mathrm{M} 9$ with $8 \mathrm{M}$ Guanidine $\mathrm{HCl}$, separated from insoluble material and the amount of protein in the supernatant was determined. The total amount of glycogen reported in the Figures was normalized relative to the total protein in the sample. All experiments were repeated at least three times and the average \pm s.d. presented in the Figures. The detailed results from individual experiments are presented in Supplementary Table 3.

Determination of reduced thiol content. Total cellular reduced thiols were quantified by reaction with $5,5^{\prime}$-dithiobis-(2-nitrobenzoic) acid) (DTNB) ${ }^{61}$. Worms were allowed to develop and grow on agar plates at $20^{\circ} \mathrm{C}$ until L4 and then transferred to NGM plates with or without glucose. For each experimental 
condition, 100 worms were collected in micro-centrifugal tubes, washed quickly 3 times with M9 buffer and incubated for $10 \mathrm{~min}$ at room temperature in M9 buffer with or without $100 \mathrm{mM}$ diamide. All animals survive such short treatment with diamide. Worms were washed three times to remove diamide. After removing most of the liquid, the worms were flash frozen in liquid nitrogen. For analysis, $\sim 150 \mu \mathrm{l}$ of M9 buffer with $2 \mathrm{mM}$ DTNB was added to the tubes with worms, frozen animals grinded with a disposable pestle and lysed by two freeze-thaw cycles. Insoluble materials were removed by centrifugation and lysates were clarified using a $10 \mathrm{kDA}$ MWCO spin filter. Absorbance at $412 \mathrm{~nm}$ was determined in the flow-through. Thiol concentration in the samples was calculated according to a standard curve generated by reaction of DTNB with GSH and then adjusted to a protein concentration in the lysates.

Fluorescent reporter assays. Worms expressing GFP under control of sod-3 (CF1553 and CF1580) were fed on NGM plates seeded with E. coli HT115 harbouring either an empty vector or a plasmid-expressing double-stranded $g s y$-1 RNAi. One (A1) and 3 (A3) day old adult worms were anaesthetized in a drop of $2 \%$ sodium azide and images were captured immediately using a Zeiss AxioZoom v16 microscope equipped for fluorescence illumination. Fluorescence intensity was quantified using the Zeiss ZEN software package.

Glycogen staining in vivo. To visualize glycogen, live worms were stained over a can with iodine as described in ref. 62. A few worms from control and glucosesupplemented plate were quickly transferred to a fresh plate, which was immediately inverted and placed over a $100 \mathrm{~g}$ bottle of iodine for $2 \mathrm{~min}$. Images were produced with a fixed exposure time using a Zeiss Discovery stereomicroscope equipped with colour camera (Optixcam). The mirror on the illuminator stage was adjusted to maximize the worm's transparency for better visualization of glycogen.

Lipid staining. Oil-Red-O staining was performed essentially as described in ref. 9. Three-day-old worms were picked, washed with M9 and fixed in 60\% isopropanol in PBS. Oil-Red-O stock (Sigma, O1391) was diluted with water to $60 \%$ and the precipitate was removed by $0.2 \mu \mathrm{m}$ filter. Worms were stained for $20 \mathrm{~min}$ at room temperature, washed with M9+0.5\% triton X100 and placed on NGM plates and images were captured using a Zeiss AxioZoom v16 microscope.

ATP content. ATP Colorimetric/Fluorometric Assay Kit (Sigma, MAK190-1KT) was used to determine ATP concentration in worm lysates. About 100 worms were picked, washed with M9 buffer and flash frozen in liquid nitrogen. Pellets were grinded with pestles in $100 \mu \mathrm{l}$ of lysis buffer and diluted with $200 \mu \mathrm{l}$ of the lysis buffer; cells were lysed by two freeze-thaw cycles and deproteinized using a $10 \mathrm{kDA}$ MWCO spin filter. ATP concentration was measured in the flow-through.

\section{Cell culture, transfection and glycogen staining. HepG2 cells (ATCC, HB-8065)} were grown in DMEM supplemented with $10 \%(\mathrm{v} / \mathrm{v})$ foetal bovine serum and $10 \mathrm{mM}$ glutamine, $\mathrm{pH}$ 7.4. Cells were grown to $70-80 \%$ confluence in $100-\mathrm{mm}$ dishes and then transiently transfected using nlucleofection (Lonza) with siRNA-targeting GYS2 following the manufacturer's protocol. Control cells were transfected with non-targeting siRNA (SiNt). Each siRNA transfection was performed with a cocktail of two siRNAs. And two combinations were used to ensure specificity $(\mathrm{Si}(2+3)$, $\mathrm{Si}(1+3)$ ). For the list of SiRNA see Supplementary Table 5. Transfection efficiency exceeded $85 \%$. Forty-eight hours post-transfection, cells were starved for $2 \mathrm{~h}$ in foetal bovine serum-free DMEM before adding media with $12.5 \mathrm{mM}$ glucose. Twenty-four hours after low glucose treatment, the media was replaced by DMEM containing $25 \mathrm{mM}$ glucose, and recombinant human insulin was added to a final concentration of $25 \mu \mathrm{M}$.

Glycogen in hepatocytes was stained by Periodic acid-Schiff (PAS) according to the manufacturer's protocol (Sigma). PAS-stained cells were imaged using EVOS cell imaging system and images were prepared using the Image J software. Percentage of glycogen was quantified using absorbance at $594 \mathrm{~nm}$ to measure PAS intensity and by normalizing it to the intensity of nuclear stain 4,6-diamidino-2phenylindole at $350 \mathrm{~nm}$.

Cell culture oxidative stress-resistance assay. HepG2 cells were treated for 30 min with $50 \mu \mathrm{M} \mathrm{H}_{2} \mathrm{O}_{2}$. The $\mathrm{H}_{2} \mathrm{O}_{2}$-containing media was removed and replaced with fresh media at $37^{\circ} \mathrm{C}$. Cell viability was assayed after $24 \mathrm{~h}$ by measuring NucGreen Dead 488 incorporation and fluorescence intensity was normalized to Hoechst staining.

Sequencing and differential expression analysis. Worms were allowed to develop and grow on agar plates at $20^{\circ} \mathrm{C}$ until they reached stage $\mathrm{L} 4$ and then transferred to NGM plates with or without glucose and incubated for $\sim 20 \mathrm{~h}$ at $20^{\circ} \mathrm{C}$. Approximately, 300 worms were collected and washed in S-buffer, and total RNA was isolated as described in ref. 63. A TrueSeq RNA Sample Preparation Kit v2 (Illumina) was used to prepare $1 \mu \mathrm{g}$ of total RNA for RNA-seq. Three independent biological replicates were done for each experimental condition. The reference genome and annotation data for $C$. elegans (Ensembl assembly based on WS220 build) were downloaded from the Illumina website (ftp://igenome:G3nom3s4u@ussd-ftp.illumina.com/Caenorhabditis_elegans/
Ensembl/WS220/Caenorhabditis_elegans_Ensembl_WS220.tar.gz). To estimate the expression level of transcripts and test for differential expression between different experimental conditions, the Tophat/Cufflinks/Cuffdiff pipeline was used ${ }^{64}$. Briefly, the RNA-seq reads were trimmed of adapter sequences and then mapped to the C. elegans transcriptome with the Tophat software package ${ }^{65}$ using the bowtie2 aligner and default parameters. The transcripts were assembled and their abundances were estimated using the Cufflinks package ${ }^{66}$. A statistical test for differential gene expression was performed using the Cuffdiff tool in the Cufflinks package with a $q$ value $(P$ value adjusted for multiple testing) threshold of 0.05 ). Analysis and visualization of the differential expression data was performed with the $\mathrm{R}$ software package (version 2.15.1) using the cummeRbund library (version 2.0).

Data availability. RNA-seq data generated in this study have been deposited in the NCBI GEO with the accession code GSE98576.

\section{References}

1. Malik, V. S., Schulze, M. B. \& Hu, F. B. Intake of sugar-sweetened beverages and weight gain: a systematic review. Am. J. Clin. Nutr. 84, 274-288 (2006).

2. Aston, L. M. Glycaemic index and metabolic disease risk. Proc. Nutr. Soc. 65, 125-134 (2006).

3. Venn, B. J. \& Green, T. J. Glycemic index and glycemic load: measurement issues and their effect on diet-disease relationships. Eur. J. Clin. Nutr. 61(Suppl 1): S122-S131 (2007).

4. Mlekusch, W., Lamprecht, M., Ottl, K., Tillian, M. \& Reibnegger, G. A glucoserich diet shortens longevity of mice. Mech. Ageing Dev. 92, 43-51 (1996).

5. Ruff, J. S. et al. Human-relevant levels of added sugar consumption increase female mortality and lower male fitness in mice. Nat. Commun. 4, 2245 (2013).

6. Colman, R. J. et al. Caloric restriction delays disease onset and mortality in rhesus monkeys. Science 325, 201-204 (2009).

7. Fontana, L. \& Partridge, L. Promoting health and longevity through diet: from model organisms to humans. Cell 161, 106-118 (2015)

8. Libert, S. \& Guarente, L. Metabolic and neuropsychiatric effects of calorie restriction and sirtuins. Annu. Rev. Physiol. 75, 669-684 (2013).

9. Lee, D. et al. SREBP and MDT-15 protect C. elegans from glucose-induced accelerated aging by preventing accumulation of saturated fat. Genes Dev. 29, 2490-2503 (2015).

10. Schlotterer, A. et al. C. elegans as model for the study of high glucose- mediated life span reduction. Diabetes 58, 2450-2456 (2009).

11. Schulz, T. J. et al. Glucose restriction extends Caenorhabditis elegans life span by inducing mitochondrial respiration and increasing oxidative stress. Cell Metab. 6, 280-293 (2007).

12. Wilkinson, D. S., Taylor, R. C. \& Dillin, A. Analysis of aging in Caenorhabditis elegans. Methods Cell Biol. 107, 353-381 (2012).

13. Lee, S. J., Murphy, C. T. \& Kenyon, C. Glucose shortens the life span of C. elegans by downregulating DAF-16/FOXO activity and aquaporin gene expression. Cell Metab. 10, 379-391 (2009).

14. Back, P., Braeckman, B. P. \& Matthijssens, F. ROS in aging Caenorhabditis elegans: damage or signaling? Oxid. Med. Cell Longev. 2012, 608478 (2012)

15. Scudellari, M. The science myths that will not die. Nature 528, 322-325 (2015).

16. Shore, D. E. \& Ruvkun, G. A cytoprotective perspective on longevity regulation. Trends Cell Biol. 23, 409-420 (2013).

17. Ristow, M. \& Zarse, K. How increased oxidative stress promotes longevity and metabolic health: the concept of mitochondrial hormesis (mitohormesis). Exp. Gerontol. 45, 410-418 (2010).

18. Lee, S. J., Hwang, A. B. \& Kenyon, C. Inhibition of respiration extends C. elegans life span via reactive oxygen species that increase HIF-1 activity. Curr. Biol. 20, 2131-2136 (2010).

19. Schmeisser, S. et al. Mitochondrial hormesis links low-dose arsenite exposure to lifespan extension. Aging Cell 12, 508-517 (2013).

20. Van Raamsdonk, J. M. \& Hekimi, S. Superoxide dismutase is dispensable for normal animal lifespan. Proc. Natl Acad. Sci. USA 109, 5785-5790 (2012).

21. Yang, W. \& Hekimi, S. A mitochondrial superoxide signal triggers increased longevity in Caenorhabditis elegans. PLoS Biol. 8, e1000556 (2010).

22. Bjelakovic, G., Nikolova, D., Gluud, L. L., Simonetti, R. G. \& Gluud, C. Mortality in randomized trials of antioxidant supplements for primary and secondary prevention: systematic review and meta-analysis. JAMA 297, 842-857 (2007)

23. de Castro, E., Hegi de Castro, S. \& Johnson, T. E. Isolation of long-lived mutants in Caenorhabditis elegans using selection for resistance to juglone. Free Radic. Biol. Med. 37, 139-145 (2004).

24. Honda, Y. \& Honda, S. The daf-2 gene network for longevity regulates oxidative stress resistance and $\mathrm{Mn}$-superoxide dismutase gene expression in Caenorhabditis elegans. FASEB J. 13, 1385-1393 (1999).

25. Vanfleteren, J. R. Oxidative stress and ageing in Caenorhabditis elegans. Biochem. J. 292(Pt 2): 605-608 (1993).

26. Wolff, S. et al. SMK-1, an essential regulator of DAF-16-mediated longevity. Cell 124, 1039-1053 (2006) 
27. Tauffenberger, A., Vaccaro, A., Aulas, A., Vande Velde, C. \& Parker, J. A. Glucose delays age-dependent proteotoxicity. Aging Cell 11, 856-866 (2012).

28. Nomura, T., Horikawa, M., Shimamura, S., Hashimoto, T. \& Sakamoto, K. Fat accumulation in Caenorhabditis elegans is mediated by SREBP homolog SBP-1. Genes Nutr. 5, 17-27 (2010).

29. Pang, S., Lynn, D. A., Lo, J. Y., Paek, J. \& Curran, S. P. SKN-1 and Nrf2 couples proline catabolism with lipid metabolism during nutrient deprivation. Nat. Commun. 5, 5048 (2014).

30. Gusarov, I. et al. Bacterial nitric oxide extends the lifespan of C. elegans. Cell 152, 818-830 (2013).

31. Mates, J. M., Perez-Gomez, C. \& Nunez de Castro, I. Antioxidant enzymes and human diseases. Clin. Biochem. 32, 595-603 (1999).

32. Luersen, K. et al. The glutathione reductase GSR-1 determines stress tolerance and longevity in Caenorhabditis elegans. PLoS ONE 8, e60731 (2013).

33. Mize, C. E. \& Langdon, R. G. Hepatic glutathione reductase. I. Purification and general kinetic properties. J. Biol. Chem. 237, 1589-1595 (1962).

34. Bus, J. S. \& Gibson, J. E. Paraquat: model for oxidant-initiated toxicity. Environ. Health Perspect. 55, 37-46 (1984).

35. Hinson, J. A., Roberts, D. W. \& James, L. P. Mechanisms of acetaminopheninduced liver necrosis. Handb. Exp. Pharmacol. 196, 369-405 (2010).

36. Lores Arnaiz, S., Llesuy, S., Cutrin, J. C. \& Boveris, A. Oxidative stress by acute acetaminophen administration in mouse liver. Free Radic. Biol. Med. 19, 303-310 (1995)

37. Lublin, A. et al. FDA-approved drugs that protect mammalian neurons from glucose toxicity slow aging dependent on cbp and protect against proteotoxicity. PLoS ONE 6, e27762 (2011).

38. Kenyon, C., Chang, J., Gensch, E., Rudner, A. \& Tabtiang, R. A C. elegans mutant that lives twice as long as wild type. Nature 366, 461-464 (1993).

39. Murphy, C. T. et al. Genes that act downstream of DAF-16 to influence the lifespan of Caenorhabditis elegans. Nature 424, 277-283 (2003).

40. McElwee, J., Bubb, K. \& Thomas, J. H. Transcriptional outputs of the Caenorhabditis elegans forkhead protein DAF-16. Aging Cell 2, 111-121 (2003).

41. Ogg, S. et al. The Fork head transcription factor DAF-16 transduces insulin-like metabolic and longevity signals in C. elegans. Nature 389, 994-999 (1997).

42. Sanchez-Blanco, A. \& Kim, S. K. Variable pathogenicity determines individual lifespan in Caenorhabditis elegans. PLoS Genet. 7, e1002047 (2011).

43. Apfeld, J., O’Connor, G., McDonagh, T., DiStefano, P. S. \& Curtis, R. The AMP-activated protein kinase AAK-2 links energy levels and insulin-like signals to lifespan in C. elegans. Genes Dev. 18, 3004-3009 (2004).

44. Narbonne, P. \& Roy, R. Inhibition of germline proliferation during C. elegans dauer development requires PTEN, LKB1 and AMPK signalling. Development 133, 611-619 (2006).

45. Curtis, R., O'Connor, G. \& DiStefano, P. S. Aging networks in Caenorhabditis elegans: AMP-activated protein kinase (aak-2) links multiple aging and metabolism pathways. Aging Cell 5, 119-126 (2006).

46. Samuelson, A. V., Carr, C. E. \& Ruvkun, G. Gene activities that mediate increased life span of C. elegans insulin-like signaling mutants. Genes Dev. 21, 2976-2994 (2007).

47. Hwang, A. B. et al. Feedback regulation via AMPK and HIF-1 mediates ROSdependent longevity in Caenorhabditis elegans. Proc. Natl Acad. Sci. USA 111, E4458-E4467 (2014).

48. McBride, A., Ghilagaber, S., Nikolaev, A. \& Hardie, D. G. The glycogen-binding domain on the AMPK beta subunit allows the kinase to act as a glycogen sensor. Cell Metab. 9, 23-34 (2009).

49. Greer, E. L. et al. An AMPK-FOXO pathway mediates longevity induced by a novel method of dietary restriction in C. elegans. Curr. Biol. 17, 1646-1656 (2007).

50. Xiao, B. et al. Structural basis for AMP binding to mammalian AMP-activated protein kinase. Nature 449, 496-500 (2007).

51. Bannasch, P. Hepatocellular glycogenosis and hepatic neoplasms. Toxicol. Pathol. 38, 1000-1002 (2010).

52. Bannasch, P. Glycogenotic hepatocellular carcinoma with glycogen-groundglass hepatocytes: a heuristically highly relevant phenotype. World J. Gastroenterol. 18, 6701-6708 (2012).

53. Floettmann, E. et al. Prolonged inhibition of glycogen phosphorylase in livers of Zucker Diabetic Fatty rats models human glycogen storage diseases. Toxicol. Pathol. 38, 393-401 (2010).

54. Laberge, A. M., Mitchell, G. A., van de Werve, G. \& Lambert, M. Long-term follow-up of a new case of liver glycogen synthase deficiency. Am. J. Med. Genet. A 120A, 19-22 (2003)
55. Weinstein, D. A., Correia, C. E., Saunders, A. C. \& Wolfsdorf, J. I. Hepatic glycogen synthase deficiency: an infrequently recognized cause of ketotic hypoglycemia. Mol. Genet. Metab. 87, 284-288 (2006).

56. Chevion, S. et al. Plasma antioxidant status and cell injury after severe physical exercise. Proc. Natl Acad. Sci. USA 100, 5119-5123 (2003).

57. Brenner, S. The genetics of Caenorhabditis elegans. Genetics 77, 71-94 (1974).

58. Apfeld, J. \& Kenyon, C. Regulation of lifespan by sensory perception in Caenorhabditis elegans. Nature 402, 804-809 (1999).

59. Dillin, A., Crawford, D. K. \& Kenyon, C. Timing requirements for insulin/IGF1 signaling in C. elegans. Science 298, 830-834 (2002).

60. Ewald, C. Y., Landis, J. N., Porter Abate, J., Murphy, C. T. \& Blackwell, T. K. Dauer-independent insulin/IGF-1-signalling implicates collagen remodelling in longevity. Nature 519, 97-101 (2015).

61. Ellman, G. L. Tissue sulfhydryl groups. Arch. Biochem. Biophys. 82, 70-77 (1959).

62. Frazier, 3rd H. N. \& Roth, M. B. Adaptive sugar provisioning controls survival of C. elegans embryos in adverse environments. Curr. Biol. 19, 859-863 (2009).

63. Reinke, V. et al. A global profile of germline gene expression in C. elegans. Mol. Cell 6, 605-616 (2000).

64. Trapnell, C. et al. Differential gene and transcript expression analysis of RNA-seq experiments with TopHat and Cufflinks. Nat. Protoc. 7, 562-578 (2012).

65. Trapnell, C., Pachter, L. \& Salzberg, S. L. TopHat: discovering splice junctions with RNA-Seq. Bioinformatics 25, 1105-1111 (2009).

66. Roberts, A., Pimentel, H., Trapnell, C. \& Pachter, L. Identification of novel transcripts in annotated genomes using RNA-Seq. Bioinformatics 27, 2325-2329 (2011).

\section{Acknowledgements}

We thank the Caenorhabditis Genetics Center (University of Wisconsin) for strains and Timur Artemyev for his contribution. This work was supported by grants from the Ministry of Education and Science of the Russian Federation grant 14.Z50.31.0014, (S.E. O.K., A.M.) Robertson Foundation and the Howard Hughes Medical Institute (E.N.)

\section{Author contributions}

I.G., A.M. and E.N. designed the experiments, analysed and interpreted the data and wrote the manuscript. I.G., O.S., B.P., L.G., I.S. and S.E. performed the experiments.

\section{Additional information}

Supplementary Information accompanies this paper at http://www.nature.com/ naturecommunications

Competing interests: The authors declare no competing financial interests.

Reprints and permission information is available online at http://npg.nature.com/ reprintsandpermissions/

How to cite this article: Gusarov, I. et al. Glycogen controls Caenorhabditis elegans lifespan and resistance to oxidative stress. Nat. Commun. 8, 15868 doi: $10.1038 /$ ncomms15868 (2017)

Publisher's note: Springer Nature remains neutral with regard to jurisdictional claims in published maps and institutional affiliations.

Open Access This article is licensed under a Creative Commons Attribution 4.0 International License, which permits use, sharing, Attribution 4.0 International License, which permits use, sharing,
adaptation, distribution and reproduction in any medium or format, as long as you give appropriate credit to the original author(s) and the source, provide a link to the Creative Commons license, and indicate if changes were made. The images or other third party material in this article are included in the article's Creative Commons license, unless indicated otherwise in a credit line to the material. If material is not included in the article's Creative Commons license and your intended use is not permitted by statutory regulation or exceeds the permitted use, you will need to obtain permission directly from the copyright holder. To view a copy of this license, visit http://creativecommons.org/ licenses/by/4.0/

(C) The Author(s) 2017 